\title{
THE AETIOLOGY, PREVENTION AND TREATMENT OF CHRONIC BRONCHITIS*
}

BY

\author{
NEVILLE SOUTHWELL \\ From Guy's Hospital
}

No one can pretend that the treatment of chronic bronchitis at the present time is anything but profoundly unsatisfactory. No attempts are made at prevention, whilst treatment of the established case depends to a large extent upon the depth of the patient's purse, and consists of a bottle of cough mixture for the poor patient, and advice to winter in South Africa for his wealthier fellow-sufferer.

It is therefore quite clear that a reconsideration of the whole problem is urgently indicated, especially as this disease is well known to be an extremely common condition in England, and one of great economic importance, extending in most cases over many years of the patient's working life, and rendering him year by year progressively less capable of following his occupation. The final result is likely to be a state of miserable invalidism, the patient himself crippled by prohibitive shortness of breath, and both he and his family irritated beyond endurance by his constant ineffectual coughing.

\section{Aetiology}

Chronic bronchitis is essentially an inflammatory condition, and to bear this constantly in mind is of cardinal importance in the understanding of the problem under discussion. In all acute and chronic inflammatory disorders the nature, severity, and duration of the disease are determined by a balance between the virulence and numbers of the causative agent on the one hand, and the local and general resistance of the patient on the other. Chronic bronchitis is no exception. The organisms are known. They are bacterial, and include micrococcus catarrhalis, pneumococci, various forms of streptococci, both haemolytic and nonhaemolytic, especially streptococcus viridans, together with Friedlander's bacillus, and staphylococci. The organisms are almost always mixed, and it is not possible to say that any of the above predominates in the majority of cases. The resistance of the patient depends upon a number of factors. It is probable that first and foremost amongst these is the natural inherited resistance of the individual. Some persons, indeed some whole families, are unduly prone to respiratory infection both of the upper and lower respiratory tract. Lowering of their general and local resistance to respiratory infection is brought about in these susceptible persons by a combination of causes; these include the obvious ones of chill, damp, fatigue, under-nutrition, and debility from a recent illness of some kind or other. No less important are the local factors: mouth breathing, excessive cigarette smoking, and any real focus of infection in the naso-pharynx. In addition, it is well known that workers in certain occupations are particularly likely to develop chronic bronchitis, and naturally these occupations are those involving the inhalation of dusts and fumes, especially those of silica, iron, steel, asbestos, carbon and kaolin; less commonly,

* A paper read before the London group of the Association of * A paper read before the London group
Industrial Medical Officers in September 1945. the fumes of chlorine, ether, ammonia, nitric acid and, strange to relate, also in a few apparently innocuous trades, such as hairdressing.

There is an additional group of cases, in which an essential underlying feature is the presence of a permanent, infective focus in the lung, usually bronchiectatic, often with fibrosis, and not uncommonly to be found in stout, thick-chested subjects with a poor respiratory movement. In this minority upper respiratory infection is of less importance in producing chronicity, but is responsible for repeated symptomatic exacerbations.

\section{Clinical Course}

It is almost invariable to find that a patient with chronic bronchitis gives a history of recurrent acute or subacute attacks in the past. In many cases the bronchial mucosa is first damaged in childhood during one or other of the acute specific fevers, most often measles or whooping cough, especially if complicated by bronchopneumonia. At that time the bronchitis clears up quickly, but probably another attack will occur the next winter, after an upper respiratory infection, and on this occasion prove more stubborn, and when the same sort of thing happens the following winter, it lingers on for many weeks, and finally, in succeeding years, tends to drag on in a mild form throughout the whole winter. The ultimate result is a permanently damaged and denuded bronchial epithelium, which reacts violently to the slightest inhaled irritant, so that the sufferer is never really free from symptoms the whole year round. Nor is this all, for owing to the obstructive element, sooner or later in most cases will appear the twin bogies of chronic bronchitis: bronchitic asthma and emphysema.

\section{Preventive Treatment}

Any attempts at prevention must be made before the chronic inflammatory changes in the bronchial tree are finally established, i.e. at a fairly early stage, when the patient is still giving a history of recurrent autumnal or winter exacerbations, but is well during the rest of the year. Every effort must then be made to protect these susceptible individuals from upper respiratory infection and its effects. This might be accomplished in various ways, and from every point of view, not least the assessment of results, the measures to be suggested could best be applied to large groups of persons rather than individuals. These measures will consist of:

1. Attempts at diminishing the frequency of upper respiratory infection in those prone to develop bronchitis.

2. Attempts at protecting the individual from the effects upon his bronchial mucosa of an upper respiratory infection, should he contract one.

It is accepted that upper respiratory infections occur either by direct droplet spread from individual to individual, or by inhalation of airborne pathogens spread in 75 
the former is probably the more common way of contracting an upper respiratory infection, the latter also accounts for a considerable number of cases, and its importance may well have been somewhat underestimated in the past.

Measures aimed at diminishing direct droplet infection have quite properly attracted a great deal of attention for a number of years past. The damage is done, of course, in crowded places, some at least of which can be avoided only with difficulty by most persons, for example the public transport vehicles. However, widespread propaganda against indiscriminate coughing and sneezing is obviously of great value, and it is evident that warning notices should be posted in all crowded places in public and industrial premises, and elsewhere. Bronchitic subjects might be well advised to walk or cycle to work if they can, or if travel is unavoidable, a bus is probably a better choice than the underground. In workrooms, proper spacing of workers is naturally essential and the erection of glass screens between opposite benches has much to recommend it. It is a wise course to send home anybody who arrives at work in the morning with a streaming head cold.

Measures aimed at controlling distant airborne droplet spread are important. Droplets are disseminated for a surprising distance by coughing, at least 15 feet, and they remain airborne for a considerable time. It has been found possible to recover influenza virus from various parts of a large room one hour after its suspension in the atmosphere. In order to prevent the spread of respiratory infection from these airborne droplets, the concentration of pathogens in the atmosphere must obviously be kept as low as possible. This might be achieved in various ways:

(i) Improved ventilation in indoor premises. Although most large buildings are equipped with some form of mechanical ventilation, natural ventilation can usually be made satisfactory by attention to various elementary points. Natural ventilation depends upon wind pressure and gravity, air being driven in through windows on the windward side and out on the leeward. Gravity effects are due to temperature differences, warm air being drawn upwards through the chimney, especially with a fire burning. If there is no chimney, roof cowls are valuable, their action being supplemented by the force of the wind itself. Windows should be adequate, the usual allocation is said to be 5 square feet of window space for every 100 square feet of floor area. Mechanical ventilation is usually of propulsion type in large premises, but extraction ventilation is quite satisfactory providing that proper inlet for air is ensured, otherwise air is drawn in through cracks and under doors, producing draughts. It is also important to remember that windows should be closed in the near vicinity of the extractor, to prevent short circuiting of fresh air. Actual air conditioning does not seem to confer any additional benefit from the point of view of ventilation. The whole subject of ventilation is admirably dealt with in a detailed article by Bedford (1944).

(ii) Bactericidal mists. Much laboratory work has been published on the use of bactericidal mists, and has shown quite definitely that the dissemination of certain mists in the atmosphere of the laboratory in small concentration is sufficient to inhibit the growth of airborne pathogens. It remains to put this method to the test of a full-scale field trial from the clinical point of view.

The essential requisite of a bactericidal mist is that it should be bactericidal. In addition, it should be quite harmless, not unpleasant in the atmosphere, noninjurious to clothing or industrial products, non-irritant to the nares, non-corrosive to metals, cheap and easy to operate. Various agents have been tried, some of them unsatisfactory as they did not fulfil the above criteria; ozone, propylene glycol and resorcins have been used, and naturally the practicability of using a penicillin mist at once comes to mind. Of the first three substances, there is much to be said for resorcin. It is genuinely bactericidal in quite small quantities in the atmosphere, and can be very easily dispersed. This is possible by electrical heating of a small metal cup containing a solid cone of resorcin, from the surface of which a gentle wisp of the mist emanates into the room noiselessly, continuously and cheaply, without requiring any attention once the apparatus is connected with a power point. Propylene glycol is also efficacious, judging from its laboratory trials, and much work has been done with it in America and in this country.

Edward, Lush and Bourdillon (1943) have shown that rapid and effective sterilization of atmospheres containing atomized particles of influenza and vaccinia viruses, and probably also herpes simplex virus, can be achieved by ultra-violet radiation of wave-length $2537 \mathrm{~A}$. At least 99 per cent. and probably more of an aerosol of influenza virus was killed by exposure for 6 seconds at a distance of $2 \mathrm{~cm}$. from either a Hanovia lamp or a G.E.C. Sterilamp. These results support the view that germicidal lamps are likely to be useful in reducing the infectivity of air contaminated with particles from persons suffering from virus infections of the respiratory tract.

Work done in the bacteriological laboratories at Guy's Hospital by F. A. Knott and the writer has demonstrated beyond a doubt the extraordinary effect of a penicillin mist in inhibiting the growth of organisms. Comparatively small quantities of the mist will inhibit growth of organisms at a considerable distance, for many hours after dispersal of the mist into the atmosphere. The passage of the mist through the apparatus does not appear to have the slightest effect upon the inhibitory action of the penicillin. It has to be remembered that the organisms are inhibited and not actually destroyed, and the addition of penicillinase will cause them to grow again. Moreover, at present the mist has to be delivered either by oxygen pressure from a cylinder, which is a costly business, or by means of an electric motor. The latter is cheap enough, and can be made noiseless by suitable enclosure. However, the solution of penicillin has to be replenished at intervals, so that the apparatus cannot be left to its own devices. It is also true that a great deal of penicillin is required to 'clear' a large room, when the apparatus is to be left running hour after hour, day after day, throughout the winter months, as would be required in any large-scale experiment. However, should large quantities of penicillin become available, it is interesting to visualize the possibility of using a mist of this kind on a controlled group of workers. It is also tempting to contemplate the possible effect of directing workers through a penicillin room on their arrival at work, bearing in mind the fact that exposure to the mist for only a few minutes appears to produce effective inhibition of bacterial organisms in the nasopharynx, an inhibition which persists for several hours.

Measures aimed at preventing the development of bronchitis in predisposed subjects who contract an upper respiratory infection must also be taken into consideration. These include both general treatment and the use of penicillin. It goes without saying that mitigation of the consequences of a head cold is to some extent a general health problem, inasmuch as the individual predisposed to bronchitis is more likely to suffer from an attack if, when he gets his cold, he is in poor physical shape, worn out by long working hours, and undernourished by the cumulative effect of some years of poor feeding. Should such a person catch a cold, as sooner or later he naturally will, then special care must be taken. He should stay at home from work, and go to bed, where he should remain for at least two days in a warm even temperature, or until his cold is better.

Various measures have been in vogue from time to time for their supposed value in limiting respiratory infection. Of these general sunlight is still popular but, although comforting, there is no real evidence that its use is successful in raising resistance to any appreciable extent. Cod liver oil has been given for the same purpose and, although here too, assessment of its value is a matter of great difficulty, it has at least stood the 
test of time, and the clinical impression of many very experienced physicians is that cod liver oil has some value in building up resistance to winter colds.

Different kinds of vaccines have been, and still are, in use as prophylactics. A stock anti-catarrh vaccine is commonly advised, but large-scale experiments carefully controlled have not shown any appreciable protection in the treated groups. It does seem, however, that the autogenous vaccine is of some value as, although it cannot be said to prevent upper respiratory infection in bronchitis, its use appears to ameliorate the severity of the symptoms of any resultant bronchitic attack, and very frequently the attacks themselves are fewer in number.

A considerable amount of investigation has now been made in the use of penicillin by spray and inhalation through the respiratory passages. Various facts emerge. Of these perhaps the most important is the observation that penicillin can be absorbed through the respiratory mucosa in sufficient amount to produce a therapeutic level in the blood, and of course very considerable quantities will then appear in the urine. Moreover, the use of sprays on the nasopharynx has a definite therapeutic value in limiting the length of infectivity of streptococcal and staphylococcal conditions there. It seems reasonable to hope that inhalations of penicillin at the beginning of a head cold may serve to prevent the onset of bronchitis in those individuals in which this is the usual sequence of events, and certainly preliminary clinical results obtained by the writer tend to strengthen this impression.

From these results it appears that no benefit is likely to follow the use of penicillin inhalations in the treatment of longstanding respiratory disease, such as established chronic bronchitis, or bronchiectasis, in which the extensive peribronchial fibrosis and poor air entry together contribute formidable obstacles to the production of any permanent improvement. In these conditions little if any clinical improvement has been noted after even a prolonged administration of penicillin over a period of some weeks, with an hour or more inhalation at a daily session, and a total dosage exceeding 3 million units. The more acute catarrhal infections, on the other hand, do appear to be a much more promising prospect, as already indicated. Although it is, of course, impossible to reach any firm conclusion based on the study of a small group of cases, it would appear justifiable to treat any head cold or upper respiratory infection in a bronchitic subject with a prophylactic course of penicillin by inhalation.

The technique of penicillin inhalation is not difficult. The apparatus used by Mutch and Rewell (1945) in their experiments in this mode of administration was an inhaler originally designed for giving adrenaline mists to asthmatics, and modified for this special purpose with specially wide-bore tubing and a closely fitting face-mask. The actual inhalation is not unpleasant at all. It is true that there is an odour somewhat reminiscent of the farmyard, but patients do not find it intolerable, and it is reassuring to know that the yellow staining of the face and moustache washes off quite easily. For the purpose of a large-scale experiment, a less extravagant apparatus is desirable, and in fact it is not difficult to produce an excellent mist with an electrically driven air current. Up to the present it has not been possible to nebulise a fine enough mist with any form of hand spray; the particles produced are much larger, and are deposited in considerable quantity in the naso-pharynx, where they are swallowed and do not reach the bronchial tree itself.

\section{Treatment of the established case of Chronic} Bronchitis

Apart from advice on general health, and the avoidance of the occupational precipitants noted earlier in this article, the duty of the physician towards the patient with real chronic bronchitis lies chiefly in the treatment of the obstinate cough, the accompanying bronchospasm, and the progressive pulmonary emphysema.

Some observers in the past have stated that no expectorant mixture is of any value at all, and have sought to prove that after administration of expectorants there is no increase in the amount of sputum produced. Whatever may be the laboratory evidence, however, all bronchitic sufferers will testify that the act of expectoration is facilitated by certain mixtures, and the distress of coughing much relieved. Not all expectorants are of equal value in this respect, and it is the strong conviction of the writer that the two of greatest worth are potassium iodide and ammonium carbonate. The former especially has a notable effect on the sputum, liquifying it and relieving bronchospasm to some extent. It should be given indefinitely in small doses throughout the winter months to these patients. To be of any use, ammonium carbonate, although unpleasant, must be given in large doses. In addition to the above drugs, the Brompton hot water mixture is often a great help in the mornings, and consists of sodium chloride, sodium bicarbonate, spirit of chloroform and aniseed water, and is given in an ounce of hot water. One other small point is the inadvisability of the common routine addition of stramonium or belladonna to these mixtures, because of their action in drying up secretions.

For the treatment of emphysema it is permissible to emphasize the surprising success of physiotherapy in some of the most unfavourable cases. Relaxation, deflation of the lungs and added mobility to the thorax, together with improved expiratory breathing power, and an increase in the vital capacity itself, can all be achieved by patient tuition in a well organized clinic, providing the subject is allowed the necessary time to attend, for it is usually many months before the maximum benefit is attained. The use of a belt to hold in the abdomen and elevate the diaphragm, thus diminishing the amount of residual air in the lungs, has been advised and is theoretically sound, but patients themselves dislike the sense of constriction round the waist and are loath to adopt the method.

Ephedrine is of great value, even in the absence of any obvious bronchospasm which, however, always exists to a certain extent in these cases. It should be given in full doses at regular intervals for an indefinite period. Some patients will complain of its side effects, such as palpitation, and it may then be found that administration as a mixture instead of as the usual tablet overcomes this drawback. It is also worth while bearing in mind that elderly bronchitics may complain of the difficulty in passing water after taking ephedrine.

\section{Conclusion}

The writer has been much impressed by the constancy of the infective factor in the great majority of cases of recurrent bronchitis. As it appears impossible at the present time to do more than ameliorate the symptoms of the chronic case, it is felt that attention should be directed towards the prevention of the preceding recurrent infective episodes. There seems to be sufficient laboratory evidence to justify the experimental use of bactericidal mists and inhalations on patients themselves.

It is suggested that it would be profitable to undertake a field trial of these methods in industry, or wherever there are large numbers of persons under continued supervision, especially where they have properly documented past medical histories.

\section{REFERENCES}

Bedford, T. (1944). Brit. J. industr. med., 1, 31.

43, 11.
Mutch, N., Rewell, R. E. (1945) Lancet, 1, 650. 